\title{
TWO-DIMENSIONAL MESH OPTIMIZATION IN THE FINITE ELEMENT METHOD
}

\author{
R. Martínez and A. Samartín
}

\begin{abstract}
The solution to the problem of finding the optimum mesh design in the finite element method with the restriction of a given number of degrees of freedom, is an interesting problem, particularly in the applications method. At present, the usual procedures introduce new degrees of freedom (remeshing) in a given mesh in order to obtain a more adequate one, from the point of view of the calculation results (errors uniformity). However, from the solution of the optimum mesh problem with a specific number of degrees of freedom some useful recommendations and criteria for the mesh construction may be drawn. For 1-D problems, namely for the simple truss and beam elements, analytical solutions have been found and they are given in this paper. For the more complex 2-D problems (plane stress and plane strain) numerical methods to obtain the optimum mesh, based on optimization procedures have to be used. The objective function, used in the minimization process, has been the total potential energy. Some examples are presented. Finally some conclusions and hints about the possible new developments of these techniques are also given.
\end{abstract}

\section{INTRODUCTION}

The finite element method (FEM) represents a wellknown procedure for efficiently solving field problems. Many computer programs based on this method are available for solving very general problems. In spite of this extraordinary development, some important problems of this method demanding further study still remain. In general, these problems are related to the quality level of the FEM results. In fact, during the past decade considerable research effort has been devoted in order to obtain some practical estimations of the error produced in FE results [1-3]. An important user's decision in relation with this error lies on the choice of the node locations in a FE mesh.

Current research is attempting to find automatic techniques to improve, in some sense, an already existing mesh by adding new degrees of freedom (new nodes or increasing the order of the polynomial in some elements). Thus a better mesh than the original is reached, because error can be nearly constant over the whole domain of the FE analysis.

In this paper, the problem of finding an optimal mesh will be considered, i.e. to find the node locations of a given topological FE mesh with a fixed number of degrees of freedom. The solution of this problem can be used to give some recommendations and guidelines for designing $\mathrm{FE}$ meshes.

This problem has been treated in the first FE error analysis. In this respect ref. [4] can be considered as a pioneer. Some results of this first research period suggest some relation between the node positions in the optimal mesh and the density of the strain energy at the node $[6,7]$. In this paper some of these conclusions are presented and other alternative techniques are hinted upon. From the results obtained recommendations for the design of the optimal mesh are also provided.

\section{1-D PROBLEMS: CLOSED-FORM SOLUTIONS}

The first group of problems that can be solved by analytical means corresponds to the mono-dimensional field problems of class $C^{0}$. The rod under axial force is a well-known example of this type of problem. In the case of a rod with constant cross-sectional properties, the optimal FE mesh obviously corresponds to the equal distance between two consecutive nodes. For this reason the next more complicated case is envisaged, namely, the rod with variable cross-sectional properties for which the optimal mesh is not known beforehand.

The most simple longitudinal variation of the cross-section corresponds to the linear one. This example will be treated below. The problem, represented in Fig. 1, to be solved is mathematically described as follows: the equation in the domain is

$$
\frac{\mathrm{d}}{\mathrm{d} x}\left(E A \frac{\mathrm{d} u}{\mathrm{~d} x}\right)=p(x), \quad \text { at } x \in(0, L) \quad[1]
$$

The essential boundary conditions are

$$
\begin{aligned}
& u=u_{1}, \quad \text { at } x=0 \\
& u=u_{2}, \quad \text { at } x=L .
\end{aligned}
$$

The natural boundary conditions are

$$
\begin{gathered}
E A \frac{\mathrm{d} u}{\mathrm{~d} x}=-p_{1}, \quad \text { at } x=0 \\
E A \frac{\mathrm{d} u}{\mathrm{~d} x}=p_{2}, \quad \text { at } x=L .
\end{gathered}
$$



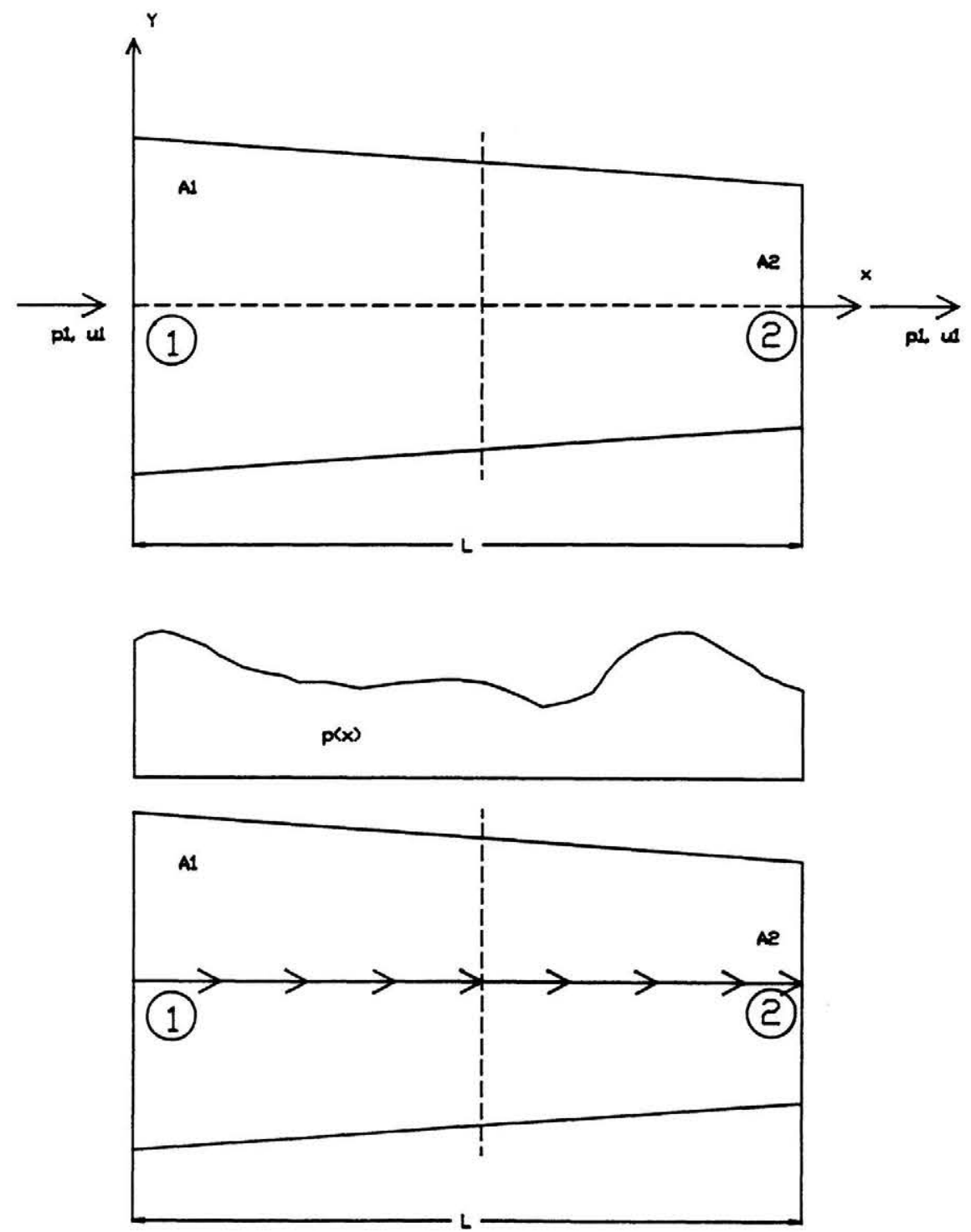

Fig. 1. One-dimensional problem.

In this particular case the linear cross-sectional variation is given by the equation

$$
A(x)=A_{1}+\frac{A_{2}-A_{1}}{L} x .
$$

The main results of this problem are summarized as follows: stiffness matrix

$$
\left|\begin{array}{l}
p_{1} \\
p_{2}
\end{array}\right|=E \frac{A}{L} \hbar\left|\begin{array}{rr}
1 & -1 \\
-1 & 1
\end{array}\right|\left|\begin{array}{l}
u_{1} \\
u_{2}
\end{array}\right| .
$$

The initial solution corresponding to the constant distributed load $p(x)=q$ (constant) along $(0, L)$ and to null displacements at the boundaries $\left(u_{1}=0\right.$, $\left.u_{2}=0\right)$

$$
\left|\begin{array}{l}
p_{1}^{0} \\
p_{2}^{0}
\end{array}\right|=q L\left|\begin{array}{c}
1-\bar{p} \\
\tilde{p}
\end{array}\right|
$$

where the constants $h$ and $p$ are defined for the expressions

$$
\begin{aligned}
& \bar{h}=\frac{2 \mu}{\ln [(1+\mu) /(1-\mu)]} \\
& \bar{p}=\frac{\mu+1}{2 \mu}-\frac{1}{\ln [(1+\mu) /(1-\mu)]}
\end{aligned}
$$

and the cross-sectional variation can be described by these two constants

$$
A=\frac{A_{1}+A_{2}}{2} \text { and } \mu=\frac{A_{1}-A_{2}}{A_{1}+A_{2}}, \quad-1 \leqslant \mu \leqslant 1 .
$$

It should be noted that the constant cross-sectional variation corresponds to the values $\mu=0$ and the situations $A_{1} / A_{2} \rightarrow \infty$ and $A_{2} / A_{1} \rightarrow \infty$ are given for the values $\mu=-1$ and +1 , respectively. The approxi- 
mate solution obtained for the above field problem by means of the FEM and using linear 1-D elements with two nodes is compared with the exact one. The comparison values are the variation of the results $h$ and $p$ with respect of the parameter $\mu$. The results corresponding to the different meshes of FE for the rod of length $L$ have been obtained in ref. [7]. They are summarized here.

1. Mesh of two nodes (one single element). The results of $h$ and $p$ are independent on $\mu$

$$
\bar{h}=1 ; \quad \bar{p}=\frac{1}{2} .
$$

2. Mesh of three nodes (two identical elements). The results are in this case

$$
\hbar=1-\frac{\mu^{2}}{4} ; \quad \bar{p}=\frac{1}{2}+\frac{\mu}{8} .
$$

The nodes are situated at $x=0, x=L / 2$ and $x=L$.

3. Mesh of three nodes (two different elements). The optimal location of the intermediate node is given by the abscissa

$$
x=\frac{L}{2}\left[1+\frac{1}{\mu}\left(\sqrt{1-\mu^{2}}-1\right)\right]
$$

and the following values are obtained for the parameters

$$
\begin{aligned}
& \bar{h}=\frac{1}{2}\left(1+\sqrt{1-\mu^{2}}\right) \\
& \bar{p}=\frac{1}{2}+\frac{1+\sqrt{1-\mu^{2}}}{4 \mu} .
\end{aligned}
$$

4. Mesh of $N$ nodes ( $N-1$ identical elements). The results are

$$
\begin{aligned}
& \bar{h}=\frac{N-1}{S} \\
& \bar{p}=\frac{1}{N-1}\left(-\frac{1}{2}+\frac{1}{S} \sum_{i=1}^{N-1} \frac{N-i}{a_{i}}\right),
\end{aligned}
$$

where

$$
\begin{aligned}
a_{i} & =\frac{1}{1-\mu+[(2 i-1) /(N-1)] \mu} \\
S & =\sum_{i=1}^{N-1} a_{i}
\end{aligned}
$$

\section{Particular cases}

For $N=2$ and $N=3$ the results already given in eqn (1) and (2) are found.

For $N=4$ (three identical elements)

$$
\hbar=1-\frac{4 \mu^{2}}{27-4 \mu^{2}}
$$

$$
\bar{p}=\frac{1}{2}+\frac{4 \mu}{27-4 \mu^{2}} .
$$

For $N=5$ (four identical elements)

$$
\begin{aligned}
& \bar{h}=1-\frac{\mu^{2}\left(5-9 \mu^{2}\right)}{16-5 \mu^{2}} \\
& \bar{p}=\frac{1}{2}+\frac{\mu\left(5-9 \mu^{2}\right)}{2\left(16-5 \mu^{2}\right)}
\end{aligned}
$$

For $N=6$ (five identical elements)

$$
\begin{aligned}
& \bar{h}=1-\frac{8 \mu^{2}\left(125-32 \mu^{2}\right)}{3125-1500 \mu^{2}+64 \mu^{4}} \\
& \bar{p}=\frac{1}{2}+\frac{4 \mu\left(125-32 \mu^{2}\right)}{3125-1500 \mu^{2}+64 \mu^{4}} .
\end{aligned}
$$

It can be checked that if $N \rightarrow \infty$ the exact results of $h$ and $p$ are reached.

5. Mesh of $N$ nodes ( $N-1$ different elements). The position of the intermediate nodes for the optimal mesh are expressed by the separations $\Delta \lambda_{i}$ between two consecutive nodes $i$ and $i+1$ (element lengths) given by the following expressions

$$
\Delta \lambda_{i}=a^{-1} \rho(1+\rho)^{i-1} \text { with } i=1,2, \ldots, N-1,
$$

where

$$
a=\frac{\mu}{1-\mu}, \quad\left(\frac{1+\mu}{1-\mu}\right)^{1 /(N-1)}-1 .
$$

The values of the rod parameters $h$ and $p$ are in this general case

$$
\begin{aligned}
& \bar{h}=\frac{[(1+\mu) /(1-\mu)]^{1 /(N-1)}+1}{[(1+\mu) /(1-\mu)]^{1 /(N-1)}-1} \frac{\mu}{N-1} \\
& \bar{p}=\frac{1}{2}+\frac{1}{2 \mu}\left[1-\frac{[(1+\mu) /(1-\mu)]^{1 /(N-1)}+1}{[(1+\mu) /(1-\mu)]^{1 /(N-1)}-1}\right] .
\end{aligned}
$$

For the particular case $N=3$ the results given in eqn (3) are again found. The exact values for $h$ and $p$ are obtained in this case when the number $N$ of nodes increases to infinity. The above results have been computed using the total potential energy of the rod as the criterium of optimization in order to find the node positions.

\section{2-D PROBLEMS: NUMERICAL SOLUTIONS}

Contrary to the 1-D problems closed form solutions are not available in this situation even for simple $C^{0}$ problems. In fact, due to highly nonlinear equations to be solved numerical procedures are needed. Mathematically the problem is stated as follows: the total potential energy is given by

$$
V=U-W,
$$


where

$$
\begin{aligned}
& U=\text { strain energy }=\frac{1}{2} \int_{V} \sigma_{i j} \epsilon_{i j} \mathrm{~d} V \\
& W=- \text { potential energy }=\int_{V} f_{i} u_{i} \mathrm{~d} V+\int_{A} p_{i} u_{i} \mathrm{~d} A
\end{aligned}
$$

$\sigma_{i j}, \epsilon_{i j}$ are the stress and strain linears components; $f_{i}$, $p_{i}$ are the body forces and the boundary pressures and $u_{i}$ are the displacement components.

The discrete FE counterpart of the above expression is

$$
V=\frac{1}{2} \mathbf{d}^{T} \mathbf{K} \mathbf{d}-\mathbf{d}^{T} \mathbf{p},
$$

where $K$ is the structure stiffness matrix, $d$ the displacements vector and $p$ the nodal forces vector. The minimum of eqn (3) is given for the displacement equations

$$
\frac{\partial V}{\partial \mathbf{d}}=\mathbf{0}, \text { i.e. } \mathbf{K d}=\mathbf{p}
$$

that produces the value

$$
V_{\min }=-\frac{1}{2} \mathbf{d}^{T} \mathbf{p}
$$

In the case that the node positions are not given, the elements of the stiffness matrix are dependent on the unknown node coordinates. Then the minimum of $V$ is obtained from the set of equations

$$
\begin{aligned}
& \frac{\partial V}{\partial \mathbf{d}}=\mathbf{0}, \quad \text { i.e. } \mathbf{K d}=\mathbf{p} \\
& \frac{\partial V}{\partial \mathbf{r}}=\mathbf{0}, \quad \text { i.e. } \mathbf{d}^{T} \frac{\partial \mathbf{K}}{\partial \mathbf{r}} \mathbf{d}-\mathbf{d}^{T} \mathbf{p}=\mathbf{0},
\end{aligned}
$$

where $r$ is the coordinates vector.

In order to solve the strongly nonlinear set of eqns (5) a numerical step by step procedure is used. At the step $i$, the current node configuration $r_{i}$ is assumed to be known, the elastic solution $d_{i}$ is obtained from the set of equations

$$
\mathbf{K}_{i} \mathbf{d}_{i}=\mathbf{p} \text {, i.e. } \mathbf{d}_{i}=\mathbf{K}_{i}^{-1} \mathbf{p},
$$

where

$$
K_{i}=K\left(r_{i}\right)
$$

The total potential energy $V_{i}$ is evaluated by the use of the expression

$$
V_{i}=\frac{1}{2} \mathbf{d}_{i} \mathbf{p}=V\left(\mathbf{r}_{i}\right)
$$

the new node configuration $r_{i+1}$ is obtained as

$$
\mathbf{r}_{i+1}=\mathbf{r}_{i}+\lambda\left(\frac{\Delta V}{\Delta \mathbf{r}}\right)_{i},
$$

where $\Delta V / \Delta r$ is an approximation of the first partial derivatives of the total potential energy at configuration $r_{i}$, with respect to the different coordinates of the nodes. The values of $(\Delta V / \Delta r)_{i}$ at configuration $r_{i}$ are obtained numerically for each $j$ component $r_{i j}$ of the coordinates vector $r_{i}$ as shown

$$
\text { component } j \text { of }\left(\frac{\Delta V}{\Delta r}\right)_{i}=\frac{V\left(r_{i}+\Delta r_{i j}\right)-V\left(r_{i}\right)}{\Delta r_{i j}}
$$

where $\Delta r_{i j}$ is the increment of the $j$ component of the vector $r_{i}$. The value of the positive parameter $\lambda$ is found by considerations of the level of accuracy required in the analysis, as it is used in the nonlinear optimization method known as the steepest gradient method.

\section{APPLICATION}

Based in the methodology shown in the previous sections a FORTRAN computer program has been written. In order to reduce the computation time some simplifications have been introduced. First, in the evaluation (7) of the first partial derivatives of the total potential energy $V$ with respect to the components of the configuration $r_{i}$ the value of $V\left(r_{i}+\Delta r_{i j}\right)$ is obtained from the expression

$$
V\left(r_{i}+\Delta r_{i j}\right)=\frac{1}{2} \mathbf{d}_{i j} p,
$$

where $d_{i j}$ is computed as an approximation solution of the equation

$$
\mathbf{K}_{i j} \mathbf{d}_{i j}=\mathbf{p}
$$

and

$$
\mathbf{K}_{i j}=\mathbf{K}\left(r_{i}+\Delta r_{i j}\right)
$$

the displacement vector $d_{i j}$ is found by the application of a simple Gauss-Seidel technique to eqn (8), assuming initial iteration values, the displacements $d_{i}$ at unperturbed configuration $r_{i}$.

A second simplification, consisted of using the general optimization program in conjunction to an automatic mesh generator, i.e. a FE preprocessor, that from a set of small number of parameters $\left(m_{1}, m_{2}, \ldots, m_{M}\right)$, the total number of node coordinates and element deformation is obtained. Thus the number of unknowns $r_{i}$ of the configuration can be drastically reduced to the number of mesh generator parameters.

\section{RESULTS}

In order to assess the efficiency and possibilities of the computer program, a simple example has been analysed. A rectangular plate of dimensions:

$\begin{array}{lc}\text { length } & 10 \mathrm{~m} \\ \text { depth } & 5 \mathrm{~m} \\ \text { thickness } & 0.1 \mathrm{~m} .\end{array}$




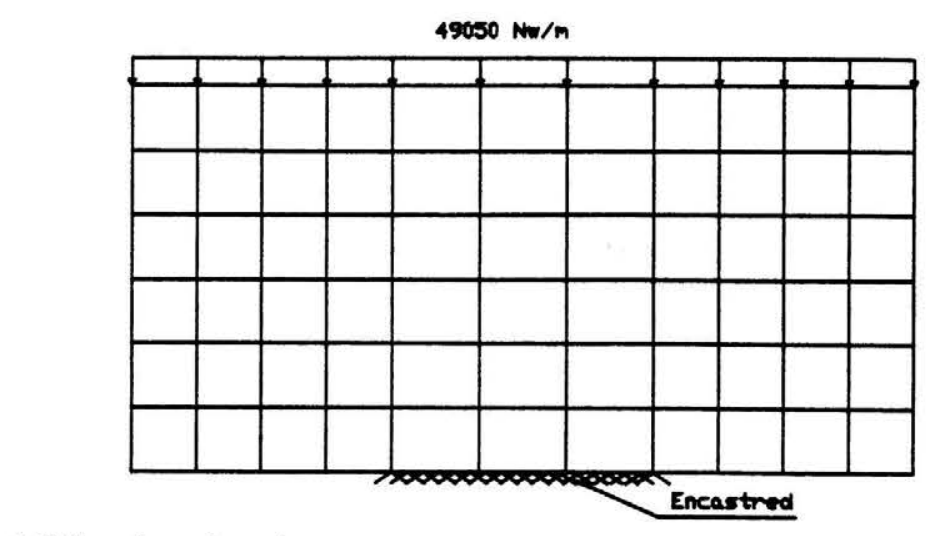

Intwal megh configuration
$C V=-40.82)$

Fig. 2. Example 1. Initial mesh.

The material constants are

$\begin{array}{lc}\text { Young's modulus } & 20.43 \mathrm{E} 09 \mathrm{NW} / \mathrm{m}^{2} \\ \text { Poisson coefficient } & 0.2 \\ \text { relative density } & 2.5 .\end{array}$

The action loads are the selfweight and a uniform distributed loading of $49,050 \mathrm{NW} / \mathrm{m}$ along the top side of the plate.

The FE discretization has been limited to have 84 nodes. As an initial configuration of nodes has been considered the one represented in Fig. 2. The corresponding total potential energy is $V=-40.22 \mathrm{~J}$. Using as unknowns the mesh generator parameters, the optimal configuration is shown in Fig. 3. The value of the total potential energy related to this configuration is $V=-48.22 \mathrm{~J}$.

In order to check the influence of the reduction of the number of unknowns, the total number of node coordinates have been used in the optimization procedure. The obtained optimal configuration is represented in Fig. 4. The total potential energy is

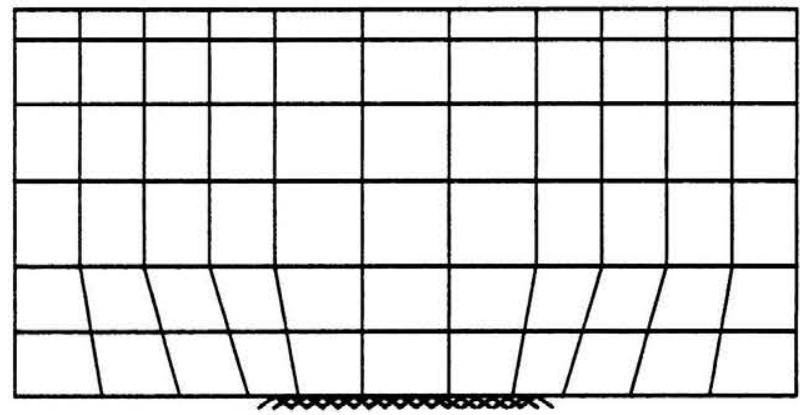

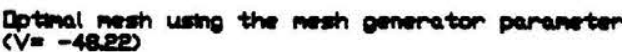

Fig. 3. Example 1. Optimal mesh using the mesh generator.

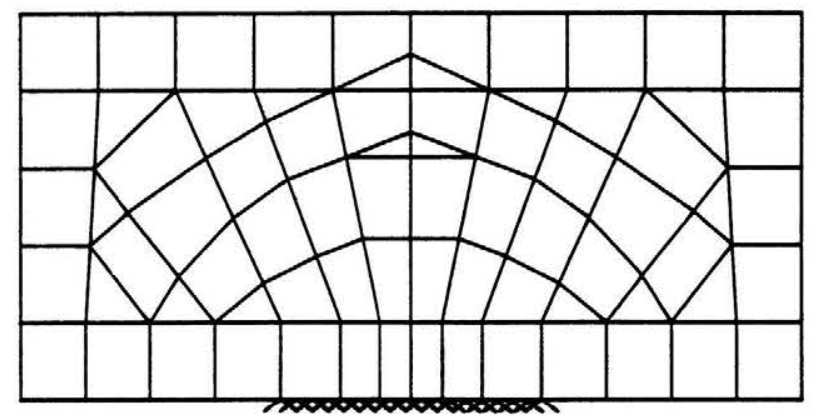

Fig. 4. Example 1. Isotactic mesh. 
Table 1. Total potential energy (Fig. 5)

\begin{tabular}{ccccc}
\hline Case & Uniform mesh & Optimal mesh & Difference (\%) & Exact value (\%) \\
\hline 1 & $-210.72 \mathrm{~J}$ & $-230.14 \mathrm{~J}$ & 9.21 & 15.56 \\
2 & $-225.33 \mathrm{~J}$ & $-255.14 \mathrm{~J}$ & 13.23 & 16.54 \\
3 & $-20.79 \mathrm{~J}$ & $-27.66 \mathrm{~J}$ & 33.04 & 18.05 \\
4 & $-94.07 \mathrm{~J}$ & $-110.05 \mathrm{~J}$ & 16.98 & 17.09 \\
5 & $-40.22 \mathrm{~J}$ & $-54.93 \mathrm{~J}$ & 36.57 & 15.79 \\
6 & $-52.05 \mathrm{~J}$ & $-58.97 \mathrm{~J}$ & 13.29 & 17.35 \\
7 & $-99.86 \mathrm{~J}$ & $-115.88 \mathrm{~J}$ & 16.04 & 13.50 \\
8 & $-100.78 \mathrm{~J}$ & $-120.78 \mathrm{~J}$ & 19.84 & 17.99 \\
\hline
\end{tabular}

$V=-54.93 \mathrm{~J}$ for this situation. From the figure it is observed that the node positions nearly follow the isostatic (principal stresses) lines.

$49050 \mathrm{Nw} / \mathrm{m}$

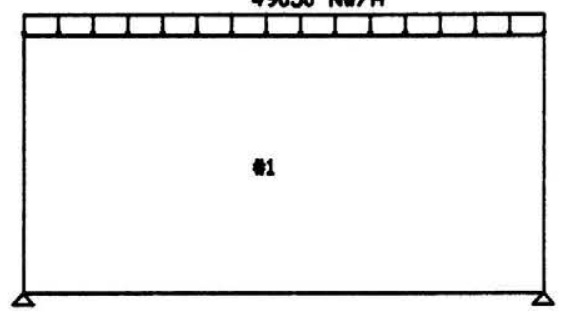

$49050 \mathrm{Nw} / \mathrm{m}$

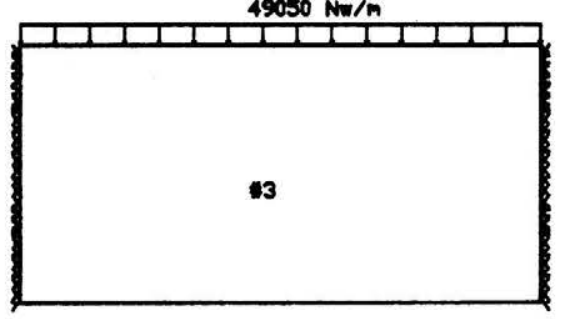

$49050 \mathrm{Nw} / \mathrm{m}$

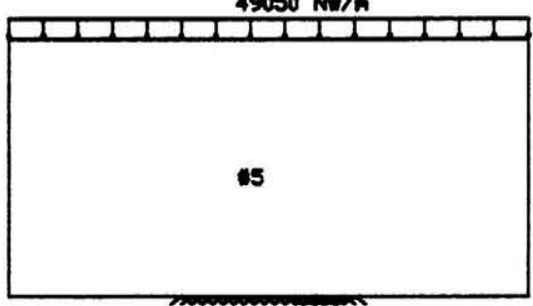

$49050 \mathrm{Nw} / \mathrm{m}$

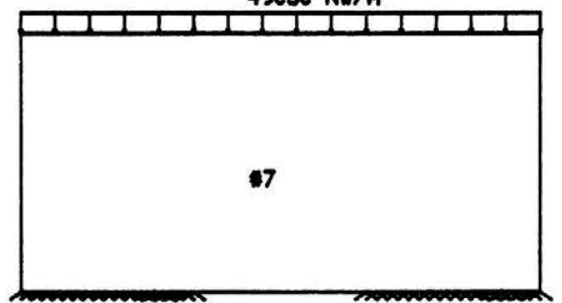

Several different cases have been analysed in order to check the optimal mesh properties. They have been represented in Fig. 5. The results obtained for these
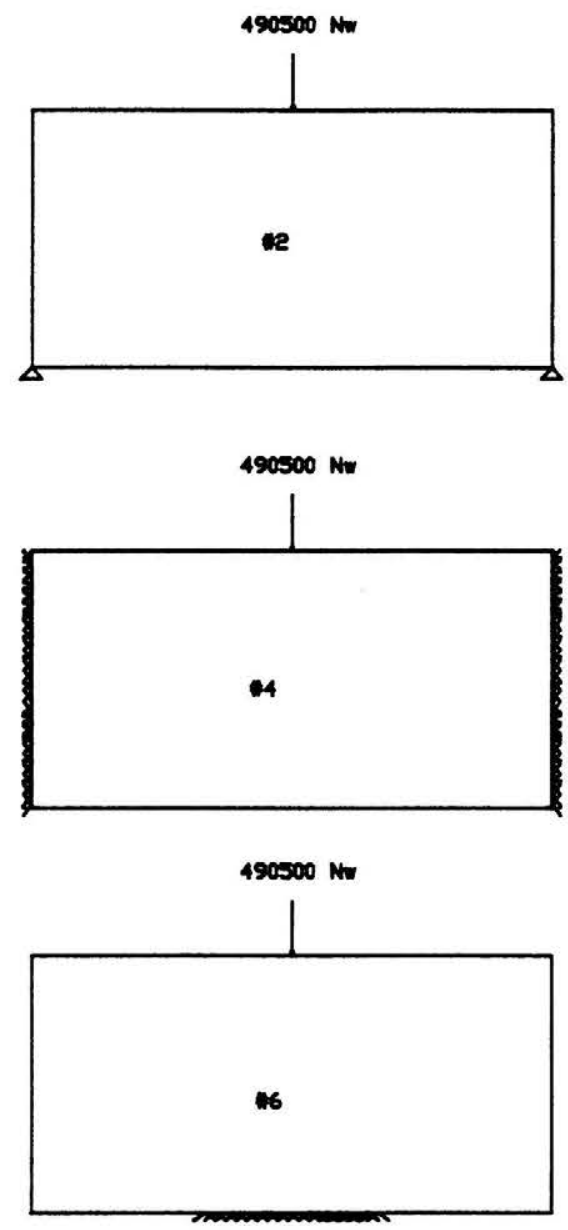

$490500 \mathrm{Nm}$

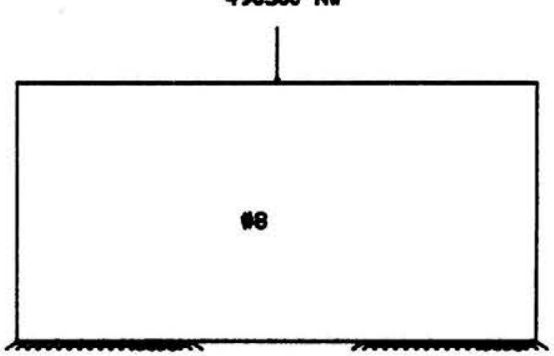

Fig. 5. Studied cases. 


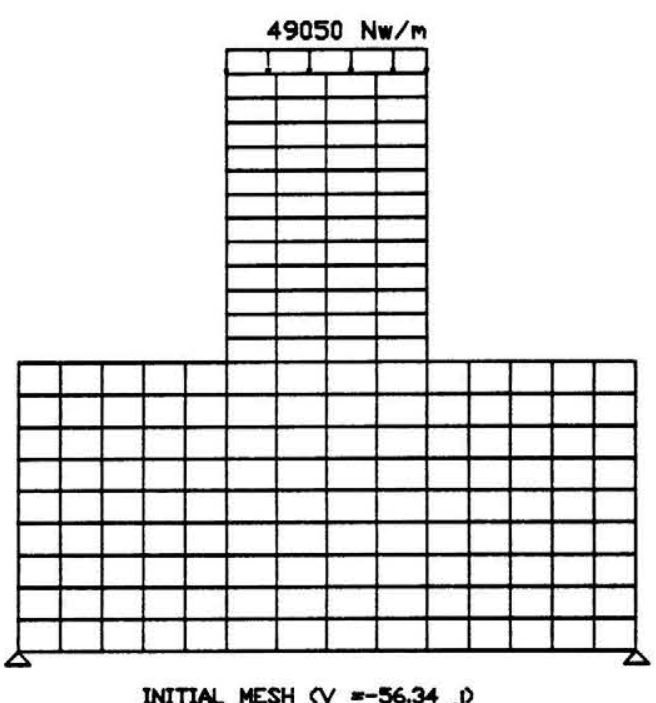

INITIAL MESH $~ V=-56.34 \mathrm{~J}$

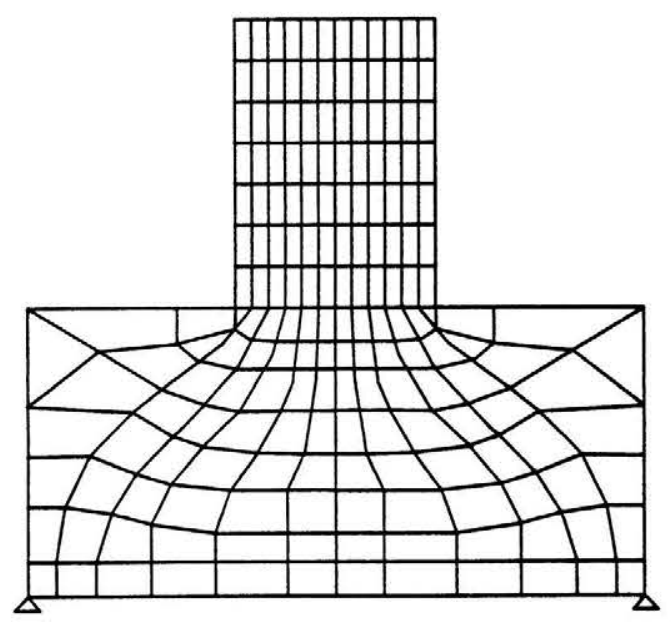

FINAL MESH $Q V=-70.23 \mathrm{~g}$

Fig. 6. Example 2.

cases are given in the table. Likewise the 'exact' values of the total potential energy of each case have been computed (using a FE analysis with more than 500 nodes) in order to assess the efficiency of these optimal meshes.
Finally in Fig. 6, a more complicated example has been studied. The initial mesh and the final mesh are shown together with the values of the corresponding total potential energy.

\section{RECOMMENDATIONS AND SUGGESTIONS}

From a large number of analysed cases some provisional recommendations about the design of FE meshes can be concluded. First, the mesh should be more dense in the areas of discontinuities (geometric, concentrated loads, large gradients of stresses, etc.). Second, it may be interesting to try to adapt the node positions along isostatic lines. This point is now under investigation in order to check its general validity. The use of a different functional to the total potential energy as a measure of the goodness of a FE mesh is currently being investigated.

\section{REFERENCES}

1. I. Babuska, The $p$ and $h-p$ versions of the finite element method. The state of the art. Inst. Phys. Sci. and Tech. Note BN-1156, University of Maryland (1986).

2. I. Babuska, O. C. Zienkiewicz, J. Gago and E. Oliveira, Accuracy Estimates and Adaptive Refinement in Finite Element Computation. John Wiley, Chichester (1986).

3. O. C. Zienkiewicz and J. Z. Zhu, Error estimates and adaptivity. The essential ingredients of engineering FEM analysis. 2nd Conference on FEM, Stratford upon Avon, May (1989).

4. B. M. McNeice and P. V. Marcal, Optimization of finite element grid based on maximum potential energy. Technical Report No. 7. University of Brown, Providence (1971).

5. D. J. Turcke and B. M. McNeice, Guidelines for selecting finite element grids based on an optimization study. Comput. Struct. 4, 499-519 (1974).

6. M. S. Shephard, R. H. Gallagher and J. F. Abel, The synthesis of near optimum Finite Element meshes with interactive computer graphics. Int. J. Numer. Meth. Engng 15, 1021-1039 (1980).

7. A. Samartin, Un estudio sobre la exactitud del método de los Elementos Finitos. Aplicación a la barra recta de sección variable bajo acciones axiles. Departamento de Análisis de las Estructuras, ETSICCP, Santander (1980).

8. J. F. Thompson, Z. V. A. Warsi and W. Mastin, Numerical grid generation. Foundations and applications. Mississippi State University (1982). 